\title{
ABSOLUTE FREQUENCY OF AN ATOMIC
HYDROGEN MASER CLOCK
}

H. E. PETERS

R. G. HALL

D. B. PERCIVAL

(NASA-TH-X-65936) ABSOLUTE FREQUENCY OF AN ATOMIC HYDROGEN MASER CLOCK H.E. Peters, et al (NASA) Jun. $19725 \mathrm{p}$ CSCL 20E

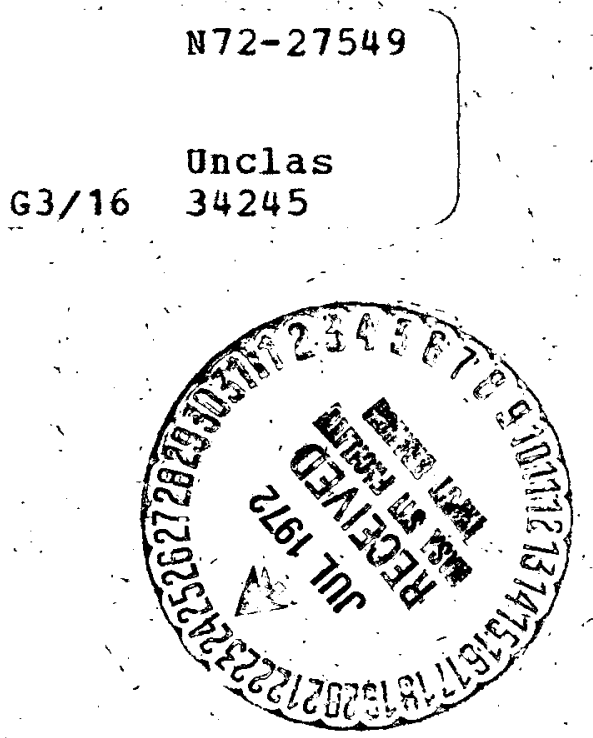

JUNE 1972 


\title{
ABSOLUTE FREQUENCY OF AN ATOMIC
}

\section{HYDROGEN MASER CLOCK}

\author{
H. E. Peters \\ NASA, Goddard Space Flight Center \\ Greenbelt, Maryland
}

and

R. G. Hall and D. B. Percival

United States Naval Observatory

Washington, D.C.

$\underline{\text { Summary }}$

An accurate determination has been made of the unperturbed atomic hydrogen ground state hyperfine transition frequency $(F=1, m=0-F=0, m=0)$ in reference to present world wide realizations of internationally defined time interval. In relation to the intermational atomic time system as derived by the Bureau International De L'Heure, the composite value is $1,420,405,751.7755 \pm 0.0031 \mathrm{HZ}$.

\section{Introduction}

The coherently synthesized integral $1 \mathrm{Mhz}$ output frequency of an atomic hydrogen maser (NP-4) developed at NASA's Goddard Space Flight Center (GSFC) ${ }^{1}$ was compared with the local atomic time scale system USNO(MEAN) derived by the United States Naval Observatory (USNO) from the mean of selected cesium standard clocks. ${ }^{2}$ The oscillation frequency of the hydrogen maser is related through determinable small correction factors to the unperturbed ground state hydrogen atom hyperfine transition frequency $(F=1, m=0-F=0, m=0)$ which we hereafter designate $f_{H}$. The USNO(MEAN) time scale is compared with the local standard frequencies of seven independent national time standards laboratories which contribute to international atomic time (IAT) as derived by the Bureau International De L'Heure $(B I H)^{3}$. The period of the measurement is February 2, 1972 to April 18, 1972.

The opportunity for the present determination arose as the result of a cooperative program between the USNO and GSFC to evaluate the long term frequency stability of the NP-4 hydrogen maser with respect to the USNO time scale system. The USNO(MEAN) scale is based upon the world's largest maintained cesium clock ensemble. The stability comparison began on January 28, 1972 when NP-4 was placed at the USNO, and it is anticipated that uninterrupted comparisons will continue through most of 1972 at the minimum.

The possibility of making the NP-4 absolute frequency measurement with regard to several international standards laboratories was realized soon after the stability comparisons began when it was observed that the errors associated with today's international time coordination links over extended measuring periods (two months in the present case) are small in relation to inaccuracy factors or reproducibility factors in presently used atomic time standards.

\section{$\underline{\text { Results }}$}

The principal results are given in Table $I$, which gives $f_{H}$ as related to the NP-4 maser with respect to the standard clock systems of the laboratories associated with the BIH.
TABLE I

Absolute Hydrogen $(F=1, m=0-F=0, m=0)$ Frequency with Respect to Time Scales of World Time Standard's Laboratories

\begin{tabular}{|c|c|c|c|c|c|c|c|}
\hline \multicolumn{8}{|c|}{$F_{\mathrm{H}}=1,420,405,751 .+.77 \mathrm{XY} \mathrm{HZ}$} \\
\hline $\begin{array}{c}F \\
\text { (France) }\end{array}$ & $\begin{array}{c}\text { USNO } \\
\text { (Wash. DC) }\end{array}$ & $\begin{array}{l}\text { IAT' } \\
\text { (BIH) }\end{array}$ & $\begin{array}{l}\text { NRC* } \\
\text { (Canada) }\end{array}$ & $\begin{array}{c}\text { PTB }^{*} \\
\text { (W. Germany }\end{array}$ & $\begin{array}{l}\text { RGO } \\
\text { (UK) }\end{array}$ & $\mid \begin{array}{c}\text { ON } \\
\text { (Swiss) }\end{array}$ & $\begin{array}{c}\text { NBS } \\
\text { (Boulder Col.) }\end{array}$ \\
\hline 0.7753 & 0.7753 & 0.7755 & 0.7755 & 0.7757 & 0.7762 & 0.7764 & 0.7772 \\
\hline \multicolumn{4}{|c|}{ Coordination Precision $0.000,08 \mathrm{~Hz}$} & \multicolumn{4}{|c|}{$\mathrm{NP}-4$ Inaccuracy $0.0024 \mathrm{~Hz}$} \\
\hline
\end{tabular}

*Maintain laboratory basic standard - see Appendix IV.

†Composite system.

The data and calculations involved in Table I are given in the Appendix. These include net absolute corrections to the NP-4 oscillation frequency of $-0.0879 \mathrm{~Hz}$ with the NP-4 associated error of $\pm 0.0024 \mathrm{~Hz}(1 \sigma)$. The value of $f_{H}$ with respect to IAT (BIH), which represents the coordinated international atomic time system, is taken as the resultant final value of $f_{H}$. The error assigned to IAT (BIH) is discussed in Appendix IV. The final results are as follows:

$$
\begin{gathered}
\mathrm{f}_{\mathrm{H}}=1420,405,751.7755 \mathrm{~Hz} \\
\sigma(\mathrm{NP}-4)= \pm 0.0024 \mathrm{~Hz} \\
\sigma(\mathrm{IAT})= \pm 0.0020 \mathrm{~Hz} .
\end{gathered}
$$

The net resultant error is taken as $\sigma^{2}=\sigma^{2}(\mathrm{NP}-4)+\sigma^{2}$ (IAT) whence $\sigma= \pm 0.0031 \mathrm{~Hz}$.

This value for $f_{H}$ is compared with other recent experimental determinations in Table II.

TABLE II

\begin{tabular}{c|c|c}
\hline \multicolumn{2}{c}{$\mathrm{f}_{\mathrm{H}}=1420,405,751 .+.7 \mathrm{XYZ} \mathrm{Hz}$} \\
\hline Laboratory (Date) & $\mathrm{f}_{\mathrm{H}}-\mathrm{Hz}$ & Inaccuracy $1 \sigma \mathrm{Hz}$ \\
\hline LSRH (69) & & \\
NASA (70) & -0.7782 & 0.0036 \\
& -0.7767 & 0.0031 \\
& & (Appendix V) \\
NBS (70) & -0.769 & 0.002 \\
NBS (70) & -0.767 & 0.002 \\
Morris (70-71) & -0.770 & 0.003 \\
IAT (Present) $^{6}$ & -0.7755 & 0.0031 \\
Paper & & \\
\hline
\end{tabular}




\section{Conclusions}

The data and results presented point to several significant conclusions.

1. The agreement of NP-4 absolute frequency with results of previous determinations is quite good (Table II). It should be noted that no specific experimental effort nor interruption of normal real time operation of $\mathrm{NP}-4$ as a clock or frequency standard was required to obtain the data.

2. The present results form the basis for later comparisons of NP-4 fre iuency and stability with regard to the USNO and to world wide standards laboratories, which will be useful in evaluating the very long term hydrogen maser performance.

3. It is possible through present world-wide time coordination links (Loran-C, Omega, Satellite transponder, moon bounce, VLF, portable clocks, etc.) to make precise time and frequency comparisons among widely dispersed international standards with a precision which exceeds present atomic standard accuracy or reproducibility by well over an order of magnitude for measuring times as short as two months. Time scale coordination on either a local or world wide basis is much more easily achievable today than absolute stability or intrinsic accuracy (Table I).

\section{Appendix}

I. Oscillation frequency of NP-4 with respect to USNO(MEAN).

For the 75 day period Feb. 2, 1972 to April 18, 1972 the operational fractional offset of NP-4 (1 MHz) with respect to USNO(MEAN) was $-10.3 \times 10^{-13} \pm 0.2 \times 10^{-13}$ (NP-4 low). The error given is the $1 \sigma$ variance $(N=2, T=5$ Days). There was no significant change in relative offset during the measurement period. The oscillation frequency, $f_{n}$, of NP-4 is related by coherent synthesis to the $1 \mathrm{MHz}$ output $\mathrm{f}_{1}$ by the relation

$$
f_{n}=N_{s} f_{1}
$$

Where $\mathrm{N}_{\mathrm{s}}=1,420.405,751,688,8$

$$
\begin{aligned}
& \text { thus } \Delta f_{n}=N_{s} \Delta f_{1}=N_{s}\left(-10.3 \times 10^{-13}\right)\left(10^{6}\right) \\
& =-0.001463 \mathrm{~Hz} \\
& \text { thus } \mathrm{f}_{\mathrm{n}}=\mathrm{N}_{\mathrm{s}}\left[\text { USNO(MEAN) }+\Delta \mathrm{f}_{1}\right] \text {. }
\end{aligned}
$$

Addition gives the oscillation frequency of NP-4: $f_{n}=1,420$, $405,751.687, \overline{3} 4 \mathrm{~Hz}$ with respect to USNO(MEAN).

\section{Absolute corrections for NP-4 oscillation frequency.}

The absolute (unperturbed) frequency $f_{H}$, of the hydrogen atom transition frequency is related to the measured value $f_{n}$ by the relation

$$
\mathrm{f}_{\mathrm{n}}=\mathrm{f}_{\mathrm{H}}+\Delta \mathrm{f}_{\mathrm{H}}+\Delta \mathrm{f}_{\mathrm{D}}+\Delta \mathrm{f}_{\mathrm{c}}+\Delta \mathrm{f}_{\mathrm{w}} \text {. }
$$

Where $\Delta f_{H}=$ Magnetic field correction

$\Delta f_{D}=$ second order doppler correction

$\Delta \mathbf{f}_{\mathrm{c}}=$ Cavity plus spin exchange correction

$\Delta \mathbf{f}_{\mathrm{w}}=$ Wall shift correction

1. Magnetic field correction.

The Zeeman frequency, $f_{z}$, of NP-4 was checked weekly. The average value was $979 \mathrm{~Hz}$ with a weckly deviation of
4. $7 \mathrm{~Hz}$ RMS. The magnetic fleld is given by

$$
\mathrm{H}=\frac{\mathrm{fz}}{1.40 \times 10^{6}}=(699.3 \pm 3.4) \times 10^{-6} \text { gauss. }
$$

The magnetic correction is given by $\Delta f_{H}=2750 \mathrm{H}^{2}$ whence it is calculated: $\Delta \mathrm{f}_{\mathrm{H}}=(0.001,345 \pm 0.000,001,3) \mathrm{Hz}$.

2. Second order doppler correction.

$\Delta f_{D}=-1.9557 \times 10^{-4} T$ where $T=$ absolute temperature of the maser bulb (atom temperature). For NP-4, $\mathrm{T}=309.1 \pm$ $0.2^{\circ} \mathrm{K}$, whence it is calculated: $\Delta \mathrm{f}_{\mathrm{D}}=(-0.060,451 \pm 0.000$, 039) $\mathrm{Hz}$.

3. Cavity and spin exchange correction.

The NP-4 maser is automatically tuned continuously ${ }^{1}$ in a manner which removes the composite cavity pulling and spin exchange correction. The NP-4 cavity correction voltage is recorded continuously. The present correction deviations in addition to previous intercomparison data on this design substantiate the following estimated error.

$$
\Delta f_{\mathrm{c}}=0 \pm 5 \times 10^{-14}(\mathrm{Max}) \text {. }
$$

4. Wall shift correction.

The wall shift parameters used in calculations of corrections and errors have been substantiated by several unpublished intercomparisons of NASA (GSFC) designs and early Varian Associates $\mathrm{H}-10$ designs. All five GSFC $\mathrm{H}-\mathrm{masers}$ as well as two Varian H-10 masers give consistent frequency agreement within $1 \times 10^{-12}$ RMS. Small 5-1/2 inch diameter bulbs coated at Varian were used originally in the $\mathrm{H}-10^{\prime} \mathrm{s}$, as well as the same masers with bulbs recoated at GSFC. The NASA masers as well as the Varian masers were consistently processed with the same materials and techniques as originally used in experiments with wall shift correction at Varian Associates ${ }^{8,9}$. The wall shift equation is given by

$$
\Delta \mathrm{f}_{\mathrm{w}}=\frac{\mathrm{K}}{6} \frac{\mathrm{A}}{\mathrm{V}}[1-a(\mathrm{t}-40)]
$$

Where $A / V$ is the surface to volume ratio for the maser storage bulb, $\mathrm{K}=0.208 \mathrm{~Hz}-\mathrm{in}, a=5 \times 10^{-3}$ and $t$ is the bulb temperature in degrees Celsius. For NP-4 the bulb is a 5. 549 liter cylinder with hemispherical ends. The inside diameter is $14.14 \mathrm{~cm}$, and the length of straight section is $25.8 \mathrm{~cm}$. The surface to volume ratio is calculated from these figures to be:

$$
\mathrm{A} / \mathrm{V}=0.8147 \mathrm{in}^{-1}
$$

Using $t=35.9^{\circ} \mathrm{C}$ and the above figures it is found:

$$
\Delta \mathrm{f}_{\mathrm{w}}=(-0.02883 \pm 0.00241) \mathrm{Hz}
$$

The net error given is the root sum square of $1 \times 10^{-12}$, the statistical deviation among individual masers, plus $1.2 \mathrm{x}$ $10^{-12^{*}}$, the estimated maximum error in the original wall shift experiments. This results in $1.6 \times 10^{-12}$ assigned wall shift uncertainty.

\footnotetext{
*The original published uncertainty was $0.43 \times 10^{-12}$, but this has been increased to the stated value upon the present authors judgment of originally unanticipated experimental uncertainties.
} 
5. Net maser absolute correction and absolute frequency $f_{H}$ vs USNO(MEAN).

Summing the corrections in 1 through 4 gives

$$
\begin{gathered}
\mathrm{f}_{\mathrm{n}}=\mathrm{f}_{\mathrm{H}}+\Delta \mathrm{f}(\text { Total }) \\
\Delta \mathrm{f}(\text { Total }=-0.08794 \pm 0.00241 .
\end{gathered}
$$

Here the net error is taken as the root sum square of the individual contributions, wherein the wall shift correction is the preponderant effect.

Subtracting $\Delta f$ ('Total) from $f_{n}$ gives, with respect to USNO(MEAN), $f_{H}=1,420,405,751.775,28 \mathrm{~Hz}$ with $\pm 0.00241 \mathrm{~Hz}$ uncertainty assigned to NP-4 alone. The non-significant last digit is retained for later computational purposes only.

III. USNO(MEAN) and $f_{H}$ in relation to other independent local time scales.

Table III gives time scale information between laboratories and the resultant relations with the USNO and NP-4. Interlaboratory data was taken from BIH circulars D65 (April 5, 1972) Section 4, and D66 (May 4, 1972) Section 4.
The error associated with an individual time coordination with IAT is conservatively estimated from the BIH circular data as 0.2 microseconds. Thus the net error becomes $\sqrt{2}$ larger with both end periods taken into account, or approximately 0.3 microseconds. The fractional individual coordination error is thus found to be $\pm 5.8 \times 10^{-14}$, or $\pm 0.000,082 \mathrm{~Hz}$ at the hydrogen frequency. To obtain $f_{H}$, the absolute frequency, in relation to the individual time scales, the offset between USNO and IAT in Table $I I$ was added to $\Delta f_{H}$ in the fifth data column, and these numbers were then added algebraically to $f_{H}$ (USNO)MEAN.

\section{IAT inaccuracy assessment.}

Only two national laboratories are presently using basic laboratory long beam cesium standards with published accuracy capability in the present range of interest to help generate their time scales. These are NRC (Canada), ${ }^{10}$ with an error limit of $\pm 1.5 \times 10^{-12}$, and PTB (W. Germany) ${ }^{11}$ with a stated error limit of $\pm 4.1 \times 10^{-13}$. The U.S. National Bureau of Standards began a major modification program in 1969 to improve the NBS 3. 66 meter primary frequency standard NBS III, but the new standard, which has been designated NBS V, is not yet operational, and the NBS time scale accuracy is thus specified at present as $1 \times 10^{-12}$, of which $5 \times 10^{-13}$ is allocated to the last

TABLE III

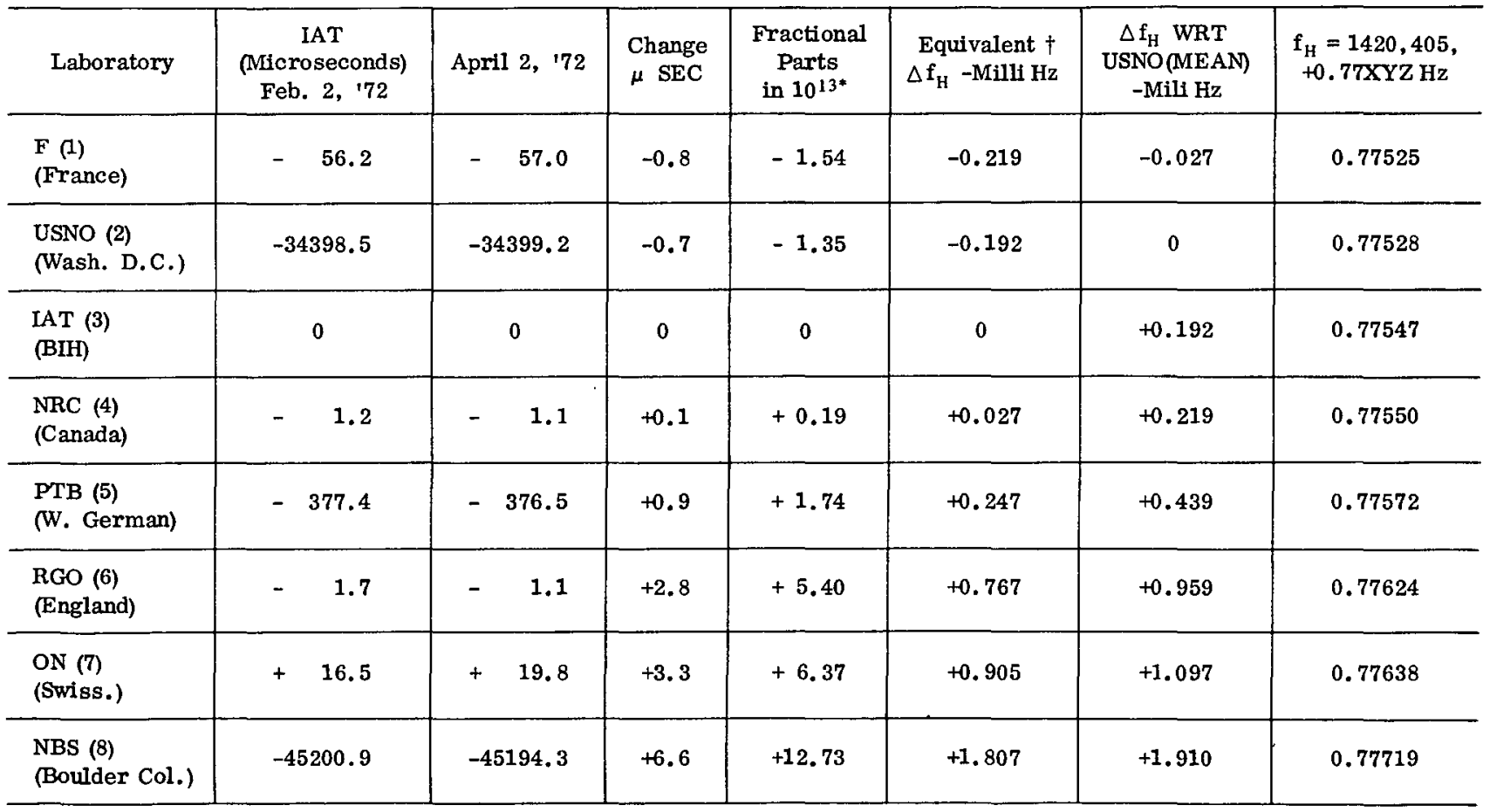

(1) Observatory of Paris, cs clock ensemble, with several cesium clocks in other locations in France, all commercial H. P., or with H.P. tubes.

(2) USNO ensemble, 14 cs clocks this measuring period, all commercial H.P.

(3) Derived TIme Scale: weights USNO $25 \%$, NBS $12.5 \%$, NRC $12.5 \%$, F $12.5 \%$, PTB $12.5 \%$, RGO $12.5 \%$, ON $12.5 \%$.

(4) NRC Ottawa, cs clock ensemble, all commercial H. P., \& 1 basic laboratory standard.

(5) PTB Braumschweig, cs clock ensemble, all commercial H. P., \& 1 basic laboratory standard.

(6) RGO Herstmonceux, cs clock ensemble, all commercial H.P.

(7) Observatory Neuchatel, cs clock ensemble, all commercial H. P.

(8) NBS Boulder, cs clock ensemble, with several cs clocks in other locations, all commercial H.P.

(*) The net elapsed time, Feb. 2 to Aprtl 2 is 60 days, or $5.184 \times 10^{6}$ seconds.

(†) Fractional change multiplied by $\mathrm{f}_{\mathrm{H}}=1.420 \times 10^{9}$. 
(1969) inaccuracy assessment of NBS $\mathrm{II}^{12}$, and the remainder to the long term instability of the NBS commercial cesium clock ensemble over the 3 year period since NBS III was operational ${ }^{13}$.

The other time scale systems relate inaccuracy primarily to the reproducibility specifications of the commercial cesium standard clocks used, which in turn have been related through manufacturers intercomparisons with house standards, or with occasional further intercomparisons with laboratory long beam cesium standards maintained by national laboratories.

In view of this complex system, the inaccuracy associated with the IAT System is arrived at as follows. First, we note that the difference between USNO and NBS is $0.0019 \mathrm{~Hz}(13.4 \mathrm{x}$ $10^{-13}$ fractionally).

The smallest inaccuracy factor for laboratory cesium beam standards officially reported to date is that of PTB (W. Germany), namely $\pm 4.1 \times 10^{-13}$.

Thus the net IAT inaccuracy factor is taken as the root sum square of $13.4 \times 10^{-13}$ and $4.1 \times 10^{-13}$ which gives

$$
\begin{aligned}
& \sigma(\text { IAT })=14.0 \times 10^{-13} \text { factionally or } \\
& \sigma(\text { IAT })=0.0020 \mathrm{~Hz} \text { at the hydrogen frequency. }
\end{aligned}
$$

The above procedure, while somewhat arbitrary, does not seem unreasonable, and is judged to give a realistic inaccuracy factor for the overall IAT System. Combining the error above with the NP-4 associated error as the root sum square gives the net error of o (Net) $= \pm 0.0031 \mathrm{~Hz}$ or $\pm 2.2 \times 10^{-12}$ fractionally.

\section{Correction of NASA (70) Data.}

Reference (5) gives the net error in the $f_{H}$ determination using the NASA(NX) hydrogen maser and five HP cesium clocks as $\sigma=0.0024 \mathrm{~Hz}$. This is judged to be optimistic. The uncertainty for wall shift given in Reference (6) for the NASA(NX) maser did not include all the uncertainty assumed herein for NP-4 wall shift correction. To put the (NX) number on the same conservative basis as the present data, the (NX) data should be corrected to give for $(\mathrm{NX})$ alone $\sigma(\mathrm{NX})= \pm 0.0024 \mathrm{~Hz}$. This may be combined with the net Cs inaccuracy, assumed in Reference (5) to be $\pm 1.33 \times 10^{-12}$ or $\sigma_{\mathrm{cs}}= \pm 0.0019 \mathrm{~Hz}$. Taking the root sum square of these gives $\sigma(\mathrm{Net})= \pm 0.0031 \mathrm{~Hz}$. This is the value the present authors assume in Table II.

\section{Bibliography}

1. Peters, H. E., McGunigal, T. E., Johnson, E. H., 22nd Freq. Cont. Symp. USAEC, Ft. Monmouth, N.J. (1968).

Peters, H. E., et. al. 23rd Freq. Cont. Symp. USAEC, Ft. Monmouth, N.J. (1969).

Peters, H. E., Proc. 3rd DOD PTTI Meeting NRL Wash., D.C. (1971).

2. Winkler, G. M. R., Hall, R. G., Percival, D. B., Metrologia $6,4,(1970)$.

3. BIH Annual Report 1970.

4. Menoud, C. and Racine, J., Z. Angew. Math. Physics 20, 578 (1969).

5. Chi, A. R., Major, F. G., Lavery, J. E., 24th Freq. Cont. Symp. USAEC, Ft. Monmouth, N.J. (1970).

6. Hellwig, et. al., IEEE Trans. I\&M IM-19, 200 (1970).

7. Morris, P., 25th Freq. Cont. Symp. USAEC, Ft. Monmouth, N.J. (1971).

8. Vessot, et. al., IEEE Trans. I\&M, IM-15, 4 Dec (1966).

9. Present Author (Peters) originally involved in development of said wall coating techniques and in experiments relating thereto.

10. Mungall, A. G., et. al., Metrologia $\underline{4}, 165$ (1968).

11. Becker, et. al., Colloque International de Chronometry, Paris (1969).

Also,

Becker, et. al., "The Cesium Beam Primary Frequency standard CS1 of the (PTB), Proc. seminar on Freq. standards and metrology, Univ. Laval, Quebec, Canada, (1971).

12. Glaze, D., IEEE Trans. I\&M IM-19, 156 (Aug. 1970).

13. Personal Communication, Dr. J. Barnes, NBS (June, 1972). 